\title{
Location Based Mobile Apps Development and Friend Finder
}

\author{
R. P. Karande ${ }^{1}$, Sachin Jundhare ${ }^{1}$, Dnyaneshwar Jadhav ${ }^{1}$, Akash Kasar ${ }^{1}$, Anant Chavan $^{1}$ \\ Department of Computer Engineering, NBN Sinhgad School of Engineering, Pune ${ }^{1}$
}

\begin{abstract}
NowadaysLocation Based Advertisement (LBA) has become today's world's most personal and directmarketing channel that provides customer more relevant information, personalized message, targeted offer about products and allows marketers to reach a specific target customersby means of digit advertisement. Location-based advertising (LBA) is a new form of advertising that is use to integratemobile advertising with location based services (LBS) to provide location-specific advertisements using real time location of consumer's devices. With the help of LBA, it is possible to target population at the right place and the right time. By taking benefit of consumer's real world position, location based advertising delivers relevant ads for products and services required. The application helps the registered shop owners to introduce the shop with available products to a consumer who is in close proximity to make them take those final steps to enter his store and let the consumer know what is around him with map support. The main feature of this application is for helping people who are new to that area .So that they can shop easily in nearby area only. One more additional feature is that one can find person nearby his location whose contact number is saved in contact list.so user can also take help from his friend to find required place.
\end{abstract}

Keywords: Android, GPS-Location, Friend-Finder.

\section{INTRODUCTION}

As advertisement of product is one of the vital parts of Server Model:

commerce, business organizations spend a significant The server side architecture is primarily made up of a amount of their investment for advertisement. Being an Model View Controller (MVC) framework. The integral part of the marketing media, the smartphone framework provides access to the relational database adoption and increasing mobile media consumption is server and other class objects within the server model. The accelerating the process. The increasing Real-time location database server is used to store user profile data, targeting uses location Information while delivering an ad advertisement location data, subscription services data, to a mobile user. Integration of Location in advertising merchant profile and other geographical related data. The greatly enhances the relevance of mobile advertising. It advertisement location table relates to Geographic has been demonstrated that the location targeted ads Information System (GIS) as it pertains to necessary generate considerably higher return than conventional information such as earth coordinates namely latitudes and mobile advertising. Considering these local facts and longitudes, street address, zip codes of every significance, an easy, customized, and effective solution advertisement is stored within it. This data is essentially 'Location Based Intelligent Advertisement (LIBS)' used to retrieve the relevant advertisements by comparing application has been developed. It is an android based the user's geographical information. The framework uses mobile application that is mainly designed to advertise the Active Records to make logical decisions. It also handles restaurants, fast-food shops, fashion houses, etc. by table associations, transactions, validations and finding the current location of the user using mapping relationship between objects and databases. The active support. The software can be described as a two way record access data from database by retrieving and communication. From user's perspective, they can find the wrapping them into a class object.

advertisement advertised by nearby shops. This real time advertising facility helps them to save time in shopping. On the other hand, shop owners have the flexibility of updating their product information any time. So consumers can get latest information about any product through advertisement.

\section{SYSTEM ARCHITECTURE}

The system architecture is explained including server model, client model and the custom built Ad Location API module.

\section{Client Model:}

The client model mainly comprises of the client-user and the GPS enabled device. The client model incorporates a mobile application program that handles all services such as requests from various APIs including geographic data from the GPS through Location Fused API module. The advantage of such an API is tremendous because no specific software application or program is needed to access GPS information at the client side. The existence of Location fused API helps the developer to concentrate more on building the advertisement delivery system within 
the client model. All the developer has to do is to make a call for the Location Fused API to get user's current location by defining the appropriate parameters such as timeout (how long API should search for location fix), interval and priority (source).

\section{Ad Location API Module:}

The system architecture also includes a custom build API which acts like an interface between the server and client model. The Ad Location API is made to run at the server side parallel to the central server program. It belongs to a class of web APIs known as Representation State Transfer (REST) APIs. REST APIs functions by using simple HTTP requests. The API is built using MVC framework similar to the server program. The API and the server program are both commonly connected to the server database. The API is delegated to perform necessary function such as retrieving advertisements from the server database.

\section{EUCLIDEAN ALGORITHM}

The Euclidean distance between point's $\mathbf{p}$ and $\mathbf{q}$ is the length of the line segment connecting bothpq. In Cartesian coordinates, if $\mathbf{p}=\left(\mathrm{p}_{1}, \mathrm{p}_{2}, \ldots, \mathrm{p}_{\mathrm{n}}\right)$ and $\mathbf{q}=\left(\mathrm{q}_{1}, \mathrm{q}_{2}, \ldots, \mathrm{q}_{\mathrm{n}}\right)$ are two points in Euclidean $\mathrm{n}$-space, then the distance (d) from $\mathbf{p}$ to $\mathbf{q}$, or from $\mathbf{q}$ to $\mathbf{p}$ is given by the Pythagorean formula:

$$
\begin{aligned}
\mathrm{d}(\mathbf{p}, \mathbf{q})=\mathrm{d}(\mathbf{q}, \mathbf{p}) & =\sqrt{\left(q_{1}-p_{1}\right)^{2}+\left(q_{2}-p_{2}\right)^{2}+\cdots+\left(q_{n}-p_{n}\right)^{2}} \\
& =\sqrt{\sum_{i=1}^{n}\left(q_{i}-p_{i}\right)^{2}} .
\end{aligned}
$$

The position of a point in a Euclidean n-space is a Euclidean vectors. So, $\mathrm{p}$ and $\mathrm{q}$ are Euclidean vectors, starting from the origin of the space, and their tips are used to indicate two points.

$$
\|\mathbf{p}\|=\sqrt{p_{1}^{2}+p_{2}^{2}+\cdots+p_{n}^{2}}=\sqrt{\mathbf{p} \cdot \mathbf{p}}
$$

\section{LBS COMPONENTS}

All In order to make LBS services possible, some infrastructure elements are necessary, including mobile devices, applications, communication network, positioning component, and the service servers Mobile devices are the tools used by users to access LBS services, to send requests and retrieve results. Such devices can be portable navigation devices (PNDs), Personal Data Assistants i.e. PDA's, laptops, mobile phones, and so on. Application is the interface for users to access the LBS service. It is usually software developed by an application provider, the downloaded and installed on the user's mobile device. A specific application is usually developed for a specific
LBS service. Due to the restrictions on mobile devices (i.e.small screen size, limited processor power and memory, and also battery capacity), LBS applications need to be lightweight and battery saving. The Communication network refers to the mobile network which is used to transferservice request from user to service provider, and requested information back to the user.Apositioning component is usually needed in a LBS application to determine the location of user's mobile device. Service providers can also maintain service servers which offer different kinds of LBS services to users and also are responsible for processing service requests and sending the back request results. Servers calculate positions, search for a route, or search specific information based on user's position. Service providers usually do not store and maintain all the information requested by users. Instead of that content providers are responsible for collection and storage of geographic data, location-based information, and related data. These data will be requested and processed by service servers and then returned to users. Figure 1 shows the interactions among those components and the process of a Location Based Service. First, user sends a service request using the application running on mobile device (Step 1). The service requested with user's current location information obtained from the positioning component i.e. GPS data, itis sent to service server via the mobile communication network (Step 2). The service server requests the service from geographic database and also other related database to get required information (Step 3, 4).At lastthe requested information is sent back to user's mobile phone through mobile communication network and the paragraphs must be indented.

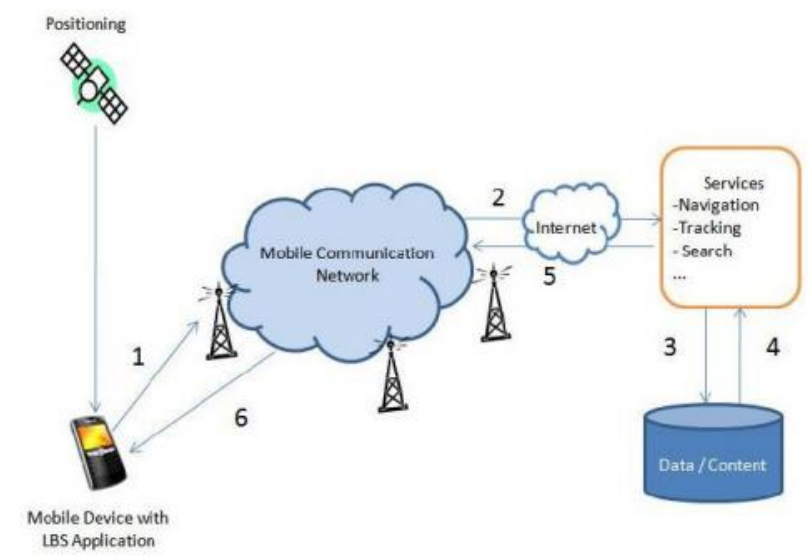

Figure 1 LBS components and Service Process

Every LBSs contains a number of components including maps and Geographic Information System i.e. GISinformation, thelocation collection services, and the LBS application-specific sub-components. Thearchitecture of LBS can be generalized as follows like Figure2.

\section{LBS Application}

LBSis used to represent a specific application such as findmy friend's application. This consists of a Smartphone components which has a number of sensors and potentially 
Vol. 5, Issue 12, December 2016

a server components that includes application specific data such as location tagged information.

\section{LBS Middleware}

This wraps access to Core LBS Features i.e.Location Tracking, GIS Provider and Location Collection Servicesto provide a consistent interface to the LBS applications.

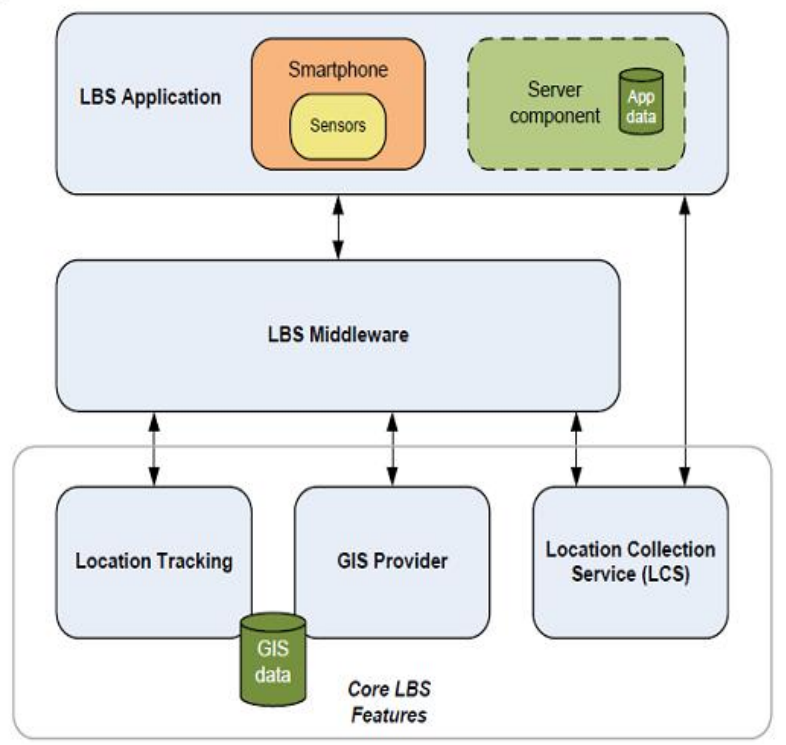

Figure 2 Components of LBS

\section{Location Tracking}

This component stores the location trace of the individual users. This represents a fundamental component in next generation ofLBS as it contains the data that allows a user's route to be determined and also to be potentially predicted. In particular, this component would typically support the following functionality: 1 . Keep records on the user's current and the past locations. 2. Also notify other components when a specific user has moved, or when they move in or out of an area. This supports location-based notifications being sent to users.

\section{GIS PROVIDER}

This component are used toprovide geospatial functionality for many LBSs including map information, map visualization and directory services. Google Maps with its API can be considered a GIS provider.

\section{Location Collection Service:}

This component are used toperform location collection to get a latitude and longitude for a specific user. Depending on the technology implemented, this component may be accessed via the LBS Middleware (For e.g., mobile network triangulation via a service provider) or directly (e.g., via GPS receiver in the Smartphone). The Android provides access to the above components to facilitate the implementation of LBS servicesthrough the help of the following classes;

\section{Location Manager \\ 2. Location Provider \\ 3. Geo-coding \\ 4. Google-Map}

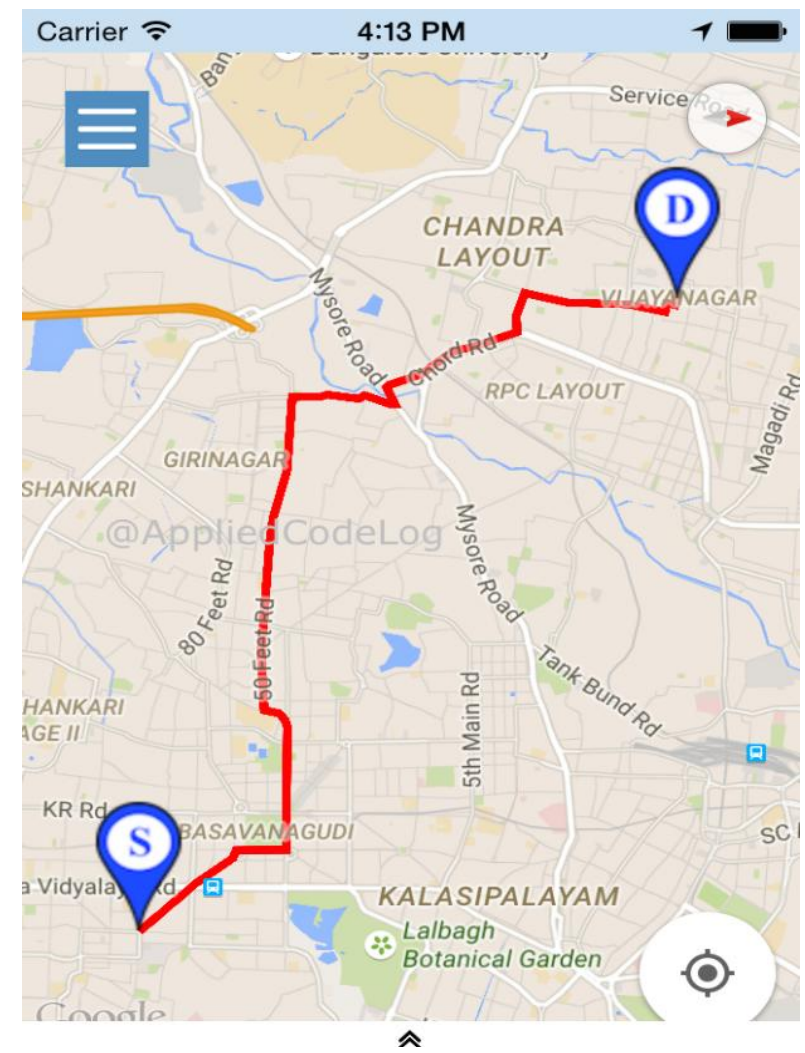

Figure3. Google map path from user location to shop location

\section{CONCLUSION}

Location based advertising has the potential to revolutionize the way advertisements are disseminated to individuals. Nowadays with enough network-bandwidth and speed advertisers can push digital advertisements with ease thereby providing a platform for two way interaction with the consumers.

Recent advances in mobile cellular networks such as $3 \mathrm{G}$, 4G LTE and WiMAX has allowed advertisement campaigners to shift their focus on mobile devices. The dubiety of smartphone Enables advertisers to target consumers based on their individual profile, location and time. In this paper we have tried to propose a location based mobile advertising system based on how frequently a user travels to a particular location.

The system can target people commuting from one location to another on a daily basis. This creates awareness among the commuters about the advertisement and offers along the approximate route they take to commute, and user can find person nearby his location whose contact number is saved in contact list.so user can also take help from his friend to find required place 


\section{REFERENCES}

[1]. Matthew A. Guido, Christopher Dougherty, Roy Casino: Geographic DatabaseOrganization that facilitates Location Based Advertising. United StatesPatent, Patent No: US 7089264 B1

[2]. JianxioHao, Zhiying Jin, DahaiRen, and LazarKoyfman: LocationBased Advertising.United States Patent Application publication, Publication No.:US2008/0027799A1

[3]. HengXu, Lih-Bin Oh, and Hock-HaiTeo: Perceived effectiveness of text vs. Multimedia Location-Based Advertising messaging. In International Journal of Mobile Communications, Interscience Publishers, Volume 7, Number2/2009

[4]. Lauri Aalto, NiclasGothlin, JaniKorhonen, TimoOjala: Bluetooth andWAP push based location-aware mobile advertising system. Proceedings of the 2nd international conference on Mobile systems, applications, and services (MobiSys 04).

[5]. Info160,http://vc4africa.biz/bLog/2012/01/10/Info160- location basedadvertisingvia-sms-in-Kenya/Android Market News, http://singularityhub .com/2011/08/16/80 android-phone-sells-likehotcakes-in-Kenya-the-world-next/.

[6]. Schwinger, W., Grin, C., Prll1, B., and Retschitzegger, W. A lightweight framework for location-based services. In Lecture Notes in Computer Science (Berlin, 2005), Springer, pp. 206_210.

[7]. Sandeep Kumar, Mohammed Abdul Qadeer, archana Gupta, Location Based Services using Android, IEEE 2009.

[8]. Location Manager APIs- Android Developer http://developer. android.com/reference/android/location/LocationManager.html 